\title{
AN EFFICIENT AND SECURE OPTIMIZED LINK STATE ROUTING PROTOCOL WITH FIREFLY ALGORITHM FOR SECURE PACKET TRANSMISSION
}

\author{
C.DANIEL NESA KUMAR ${ }^{\# 1}$, Dr. V. SARA VANAN ${ }^{\# 2}$ \\ ${ }^{\# 1}$ Ph.D-Research Scholar, Department of Computer Science, Hindusthan College of Arts and Science, \\ Coimbatore, Tamil Nadu, India \\ ${ }^{\# 2}$ Professor and Head, Department of Information Technology(PG), Hindusthan College of Arts and \\ Science, Coimbatore, Tamil Nadu, India
}

\section{ABSTRACT}

A Mobile Adhoc Network (MANET) includes a set of independent mobile nodes which communicates with each other by means of radio waves. The construction of nodes depends on the network actions and hence MANTE has a dynamic shape and a limited bandwidth with complete wireless connectivity. As MANETs are dynamic, the network topology would change often. Hence, it is important to frequently update the network information at each and every node for achieving an efficient data routing. The design of routing protocol with energy efficiency and security is a demanding task. To overcome this task, the previous system designed an energy-efficient secured routing protocol called as Secure Optimized Link State Routing (SOLSR) protocol. However it does not efficiently select the Multipoint Relays (MPRs) to forward control messages. In order to solve this problem the proposed system designs an efficient Secure Optimized Link State Routing (SOLSR) Protocol with Firefly Algorithm (FA) for secure packet transmission. In this protocol, the FA is used to optimally select the MPRs with the help of packet delay and Packet Delivery Ratio (PDR) of the node. After choosing the link, the power statuses of the nodes are checked and routes are established. Finally compute group key distribution using the generated keys. In order to avoid non authorised nodes and use of the same group key in more than some amount of data, the group key is changed regularly. Finally the secure Source Anonymous Message Authentication Scheme (SAMA) is utilized to provide the communication privacy. The experimental results show that the proposed system achieves better performance compared with the existing system in terms of PDR, energy consumption and end to end delay.

Key words: Mobile Adhoc Network (MANET), routing, Firefly Algorithm (FA) and authentication

\section{Corresponding Author: C. Daniel Nesa Kumar}


DOI: https://dx.doi.org/10.26808/rs.ca.i8v2.11 International Journal of Computer Application (2250-1797)

Issue 8 Volume 2, March-April 2018

\section{INTRODUCTION}

A Mobile Adhoc Network is a group of autonomous mobile nodes which can communicate with one another through radio waves. The mobile nodes which are in radio range of one another can communicate directly, while the rest require the assistance of intermediate nodes for routing their packets [1]-[3]. Every one of the nodes is equipped with a wireless interface for communicating with one another. These networks are distributed fully, and can operate at any place with no support of any pre-determined infrastructure in the form of access points [4]-[5].

A routing protocol indicates the means in which the routers communicate with one another, distributing information which facilitates them to choose the routes between any two nodes over a computer network. Thus the routing algorithms decide the particular selection of route [6]. Every router is rendered with a prior knowledge about the networks directly connected to it. A routing protocol takes this information first among the next immediate neighbors, and thereafter through the entire network. By this means, routers come to know regarding the network topology.

The working of secure routing has a significant role to play in MANETs security because of the lack of fixed infrastructure. Data transformation in the case of ad hoc network has to be carried out in a secured manner. The security challenges in ad hoc network are dynamic topology, bandwidth, smaller device size and less battery life. Owing to the dynamic nature, it is hard to have secured transmission maintained in the network [7].

The ad hoc network does not rely on any pre-available infrastructure such that the node can go out and then join the network in a situation where the security may fail. Two kinds of attack happen in ad hoc network, first one is passive attack, and this attack does not modify the transmitted information in the network. But, it can permit unauthenticated users to find the message. Second one is active attack, which is a severe one and disrupts the message flow between the nodes present in the network. It may permit the unauthenticated user to make modifications to the message. The adversary node can be recognized by means of dropped packets, drained battery, bandwidth consumption, unreliable packets, delay, connection failure and false routing [8].

\section{LITERATURE SURVEY}

Nikhil et al., (2012), used GA for intrusion detection. Static shortest path (SP) issue has been well dealt with employing smart optimization techniques, e.g., artificial neural networks, genetic algorithms (GAs), particle swarm optimization, etc. But, with the development in wireless communications, more number of mobile wireless networks have made their appearance, e.g., Mobile Ad Hoc Networks (MANETs), wireless sensor networks, etc. One of the most significant features in mobile wireless networks is the topology dynamics, i.e., the network topology varies over time because of the energy conservation or node mobility. Hence, the SP routing issue in MANETs transforms to be a dynamic optimization issue. GA's are capable of finding, at least an optimal path, even if not the shortest, between the source and destination in mobile ad-hoc network nodes. And the alternate path or backup path to prevent there route discovery in link failure or node failure [9].

Albers et al., (2002), introduced a distributed and cooperative architecture of IDS by employing mobile agents. A Local Intrusion Detection System (LIDS) is realized on each node for local concern that also can be extended for the purpose of global concern by collaborating with other LIDS. Two kinds of data are exchanged among the LIDS: security data (for obtaining complementary information from cooperating nodes) and intrusion alerts (to warn others regarding locally detected intrusion). In order to evaluate the probable intrusion, data has to be got from what is detected by the LIDS, besides the additional information from the rest of the nodes. Other LIDS may be executed on various operating systems or make use of data from diverse activities like system, application, or network activities; hence, the format of this raw data may be diverse that renders it difficult for LIDS to 
DOI: https://dx.doi.org/10.26808/rs.ca.i8v2.11 International Journal of Computer Application (2250-1797)

Issue 8 Volume 2, March-April 2018

carry out the analysis. But, such challenges could be resolved by employing SNMP (Simple Network Management Protocol) data which is located in MIBs (Management Information Base) in the form of an audit data source [10].

Gupta et.al (2013) proposed a concept of trust factor in (initialization phase), for choosing the most effective route along with a routing path. In this strategy, Constant trust factor is utilized for evaluating the reliable and shortest path for communication in the network. In order to avoid the attack by malicious node, the identity information such as IP address and Trust factor value has been employed. This identity information is allocated to every node in initialization phase or at the time the node is configured [11].

Pirzada et.al (2006) transformed Dynamic Source Routing (DSR) protocol so that the intermediary nodes function as trust gateways which keep trace of trust levels of the nodes for preventing the malicious nodes. Every node monitors its neighbors and then sustains a direct trust value for them. Source node employs this trust information for computing the path that is the most trustworthy. The technique for the computation of trust is dependent only on the forwarding behavior of the nodes. The node which executes the trust gateway might itself be malicious [12].

\section{PROPOSED METHODOLOGY}

\subsection{Overview}

An Secure Optimized Link State Routing Protocol (SOLSR) with Firefly Algorithm (FA) is utilized to choose a secure and efficient link for routing.

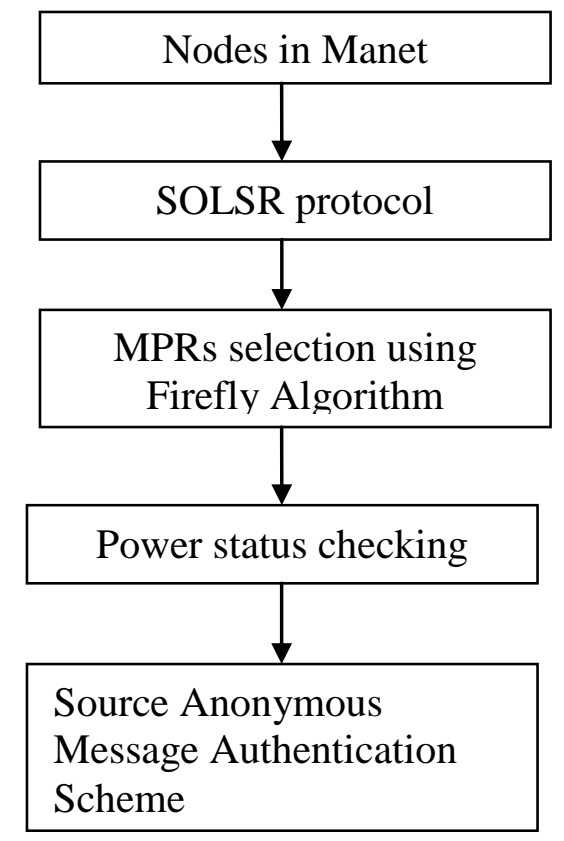

\section{Figure 1.Flow diagram of the proposed system}

In this proposed system Multipoint Relays (MPRs)are selected by using Firefly Algorithm based on the packet delay and Packet Delivery Ratio (PDR) of the neighboring nodes. The proposed system uses a minimum number of messages in the group key allocation procedure to decrease energy consumption. In this proposed protocol group key is sporadically distorted to evade non-authorized nodes and to evade the exploit of the similar group key in more than several amount of data.

Each route table has an entry for its power status. The power status of the nodes is checked by using Power Efficient Routing Scheme and a route is established. Same process is repeated in route 
DOI: https://dx.doi.org/10.26808/rs.ca.i8v2.11 International Journal of Computer Application (2250-1797)

Issue 8 Volume 2, March-April 2018

repair phase. Once the secured link is chosen this scheme will give communication privacy for both message sender and message recipient using Secure Source Anonymous Message Authentication Scheme (SAMAS). Then the designed approach will provide message content authenticity without relying on any trusted third parties.

\subsection{Secure Optimized Link State Routing (SOLSR) Protocol}

In this protocol, number of mobile nodes is jointly supporting network operation. The network is partitions into several parts. The membership is frequently changed at anytime. The group of nodes in the network is communicated through the routing protocol. The similar set of nodes must share the similar collection key for routing control message interactions. All the network nodes are responsive of the number of nodes with which they can communicate. By using Multipoint Relays (MPRs) Secure and Efficient Link Optimizated Routing Protocol controls flooding. The relays are only responsible transmit the control messages. Every node selects MRP nodes between groups of one hop neighbors as well as two hop neighbors. In this protocol nodes can able to find the appropriate delay among its clocks to avoid replay attacks. The protocol used this information for provides the weak harmonization on the network. The Access Control Entity (ACE) is assumed in this network. It forwards the alert while nodes add or removed from the network. And also it generates the record to every node which integrating with a public key $\mathrm{Ki}$ to number of identity IDi. A node is called as authorized by ACE broadcasting the node recognition ID to the network. In order to achieve group key, the ACE must sign a public key $\mathrm{Ki}$, a private key $\mathrm{ki}$ and the certificate $\mathrm{Ci}$ which is mandatory by an authorized node. The list of authorized nodes is maintained by every authorized node.

\subsubsection{Multipoint Relays (MPRs) selection using Firefly Algorithm}

The Multipoint Relays (MPRs) are utilized to forward the control messages. Each node in the network is selecting the one hop neighbour MPR nodes using Firefly Algorithm. In this work delay and packet delivery ratio are considered as an objective function.

Firefly algorithm is one among the effective optimization techniques. Firefly algorithm is dependent on over emphasizing the flashing feature of fireflies. Firefly algorithm is dependent upon two significant things, primary one is the difference in light intensity and second one is creation of attraction. For easiness it is presumed that by its vividness, attraction of firefly is identified that is related to the objective function. The ideal three rules are:-

1. Each and every fireflies are taken as unisex and one firefly is engrossed to other fireflies regardless of the sex

2. The Attraction is relative to their vividness that signifies for any two blinking fireflies, the movement of firefly is from minimum vividness in the direction of the perkier one and it would travel arbitrarily when no one is sunnier than other. Likewise, as their distance rises, they both reduce.

3. The landscape of the objective function has impact on the vividness of the firefly. The vividness is relative to the objective function's value for a maximization issue.

At specific location $\mathrm{x}$, for a maximization issue, the vividness $\mathrm{I}$ of a firefly could be selected as $\mathrm{I}(\mathrm{x}) \propto \mathrm{f}(\mathrm{x})$.

The light intensity I differs with the distance $r$ containing a fixed light absorption coefficient $\gamma$ i.e

$$
\mathrm{I}=I_{o} e^{-\gamma r^{2}}
$$


DOI: https://dx.doi.org/10.26808/rs.ca.i8v2.11 International Journal of Computer Application (2250-1797)

Issue 8 Volume 2, March-April 2018

The firefly's attraction is directly relative to the light intensity realized by other fireflies; the attraction $\beta$ with the distance $r$ id defined as

$$
\beta=\beta_{0} e^{-\gamma r^{2}}
$$

Here $\beta 0$ is the attraction at $r=0 . r_{i, j}$ is the distance amid any two fireflies $i$ and $j$, that are at positions $\mathrm{x}_{\mathrm{i}}$ and $\mathrm{x}_{\mathrm{j}}$ correspondingly. The Cartesian distance is provided by the equation

$$
r_{i j}=\left|x_{i}-x_{j}\right|=\sqrt{\sum_{k=1}^{d}\left(x_{i, k}-x_{j, k}\right)^{2}}
$$

Here $x_{i, k}$ is known as the $\mathrm{k}^{\text {th }}$ element of the spatial coordinate $\mathrm{x}_{\mathrm{i}}$ of the firefly $\mathrm{i}$ and $\mathrm{d}$ is known as the amount of dimensions. The movement of a firefly $\mathrm{i}$ in the direction of more another (brighter) firefly $\mathrm{j}$ is provided by

$$
x_{i}=x_{i}+\beta_{0} e^{-r r_{i j}^{z}}\left(x_{j}-x\right)+\alpha \varepsilon_{i}(4)
$$

For the attractiveness, Second component is utilized and for randomization, third component is utilized with $\alpha$ being the randomization parameter, and $\varepsilon_{i}$ is known as a vector of random numbers being derived from a Gaussian distribution or else uniform distribution interval $[0,1]$.

Here delay, throughput, transmission energy and Packet Delivery Ratio (PDR) are considered as an objective function. The parameters are calculated based on the weight basis and $\mathrm{P}$ denotes the packet delay, PDR denotes the packet deliver ratio.

$$
F_{i}=W_{1} * P+W_{2} * P D R
$$

Minimize Delay, $D e_{p}=\sum_{(i, j) \in E} d e_{i j} x_{i j}$

Maximize packet delivery ratio $\mathrm{PDR}=\sum_{(i, j) \in E} P D R_{i \bar{i}} x_{i j}(7)$

Where, $x_{i j} \in\{0,1\} \forall(i, j)$

$d e_{i j}$ is delay between node $(\mathrm{i}, \mathrm{j})$

$\mathrm{PDR}_{\mathrm{ij}}$ is packet delivery ratio between node $(\mathrm{i}, \mathrm{j})$

\section{Algorithm 1:}

Firefly algorithm

1. Produce initial population of fireflies ( number of nodes) $x_{i}(i=1,2, \ldots n$ )

2. Compute light intensity (PDP and delay)of fireflies

3. Describe light absorption coefficient $\gamma$

4. While $(\mathrm{t}>$ Max Generation)

5. for $\mathrm{i}=1: \mathrm{n}$ all $\mathrm{n}$ fireflies

6. for $\mathrm{j}=1: \mathrm{i}$ all $\mathrm{n}$ fireflies

7. Light intensity Ii at $x i$ is identified

8. if $(\mathrm{Ij}>\mathrm{Ii})$ 
9. Move firefly $\mathrm{i}$ in the direction of $\mathrm{j}$ in all $\mathrm{d}$ dimensions

10. Else

11. Move firefly i arbitrarily

12. End If

13. Attractiveness modifies with distance $r$ via $\exp [-\gamma \mathrm{r} 2]$

14. Identify novel solutions and revise light intensity

15. End for $\mathrm{j}$

16. End for $i$

17. Rank the fireflies and identify the present best

18. end while

19. Post process outcomes and visualization

Based on the FA, the optimal MRP node is selected for packet transmission.

\subsection{Power efficient routing}

Every route table having power status of nodes in the network. To form a new route power status of the nodes are evaluated and route is recognized. This process continued in repair phase. For each hello interval the route table has been updated. After Tn time, the energy consumption of the nodes are computed by using

$$
\mathrm{E}\left(T_{n}\right)=\mathrm{nt}^{*} \alpha+\mathrm{nr}^{*} \beta
$$

Where,

E(Tn) - energy consumed by a node after time Tn.

nt -number of packets transmitted by the node after time Tn

$\mathrm{nr}$-number of packets received by the node after time Tn.

$\alpha$ and $\beta$ - constant factor

The remaining energy $E_{\mathrm{Rem}}$ of the node at time $\mathrm{T}$ is computed by

$$
\mathrm{E}_{\mathrm{Rem}}=\mathrm{E}-\mathrm{E}(\mathrm{Tn})
$$

At first source node create a REQ with Emin set to $100 \%$. After that every neighboring nodes fix minimum energy of REQ to their power range.

Then all the intermediate nodes sets minimum energy level field of REQ to their energy level. When the REQ is obtained, the neighboring nodes overwrites least amount energy level Emin of the REQ packet if $E_{R e m}<$ Emin. Else, Emin remains unaffected. Finally the neighboring nodes send the route request to its neighbors. This procedure is continued until REQ reaches to the destination.

\subsection{Group key distribution}

The group key distribution scheme allows the substitute of the group key occasionally or when a node is expelled. The intermittent distribution rejects adversaries with the group key, except private key. To achieve the group key distribution, an Intrusion Detection System (IDS) is used. If any malicious activity is happen the IDs send the alert. The group key allocation is initiated by the round leader which distributes the declaration message to specify the continuation of a new group key. In announcement listening phase, the round leader sends the message to accept the generated group key. The leader stops the procedure when the nearest nodes send the response message. It includes new group key encrypted with its neighbor's public key. The MultiPoint Relays (MPRs) of leaders known 
DOI: https://dx.doi.org/10.26808/rs.ca.i8v2.11 International Journal of Computer Application (2250-1797)

Issue 8 Volume 2, March-April 2018

as nearest node, additionally it is used for retransmit the Announcement message, and nearest nodes by two hops select an MPR to achieve the novel group key. All network MPRs replicate this scheme so as to guarantee the all nodes greeting of the new group key

\begin{tabular}{|c|c|}
\hline 01234 & 5678901234567 \\
\hline Type & Reserved \\
\hline Sourc & \\
\hline Key S & mber \\
\hline Curre & ader \\
\hline Next & \\
\hline $\begin{array}{l}\text { Time: } \\
\text { mech }\end{array}$ & beginning of the \\
\hline Signa & vate key \\
\hline Certif & \\
\hline
\end{tabular}

Figure 2 : Announcement message

Figures 2 and 3 represent the messages. The signature, certificate, and encrypted key have a variable size according to the hash function, cryptography scheme and key size. To send the message and give the assure to content integrity, the certificate and message signature are utilized. When all the nodes provides guarantee about their originality on the authorized node list, the key allocation for each pair of nodes is unbeaten.

\begin{tabular}{|l|l|}
\hline 01234567890123456789012345678901 \\
\hline Type & Reserved \\
\hline Source ID & \\
\hline Destination ID \\
\hline Key Sequence Number \\
\hline $\begin{array}{l}\text { New Group Key Encrypted with } \\
\text { Neighbor Public key }\end{array}$ \\
\hline Signature with private key \\
\hline Certificate \\
\hline
\end{tabular}

Figure 3: Response message 
DOI: https://dx.doi.org/10.26808/rs.ca.i8v2.11 International Journal of Computer Application (2250-1797)

Issue 8 Volume 2, March-April 2018

At the same time, the nodes in the networks are establishing the new group key roughly. Hence, by utilizing the new group key Tn, every nodes compute the probable time to start.

$$
\mathrm{Tn}=\mathrm{Ta}+\mathrm{Td} \times \mathrm{H}(10)
$$

Where,

Ta - approximate time at the starting of group key allocation

Td- Computation of the highest delay taken by a MPR for broadcast the fresh group key to its nearest node.

$\mathrm{H}$ - Number of hops among the round leader and the node very distant from it in the network.

After Tn, nodes start to utilize the generated group key though they accept messages marked with older or generated group key in the interval specified by Tn - DT and Tn + DT.

Where,

DT - the delay tolerance.

After Tn + DT, the messages are does not indicated with the generated group keys are superfluous. The nodes are called as new nodes when the nodes does not accept the group key before Tn + DT. It can be achieve new group key with the joining scheme.

\subsection{Source Anonymous Message Authentication Scheme}

Once the secured link is computed, the Secure Source Anonymous Message Authentication Scheme (SAMAS) is utilized to give the communication privacy both message sender and receiver. The designed system includes consists of three steps for creating the whole SAMAS. Additionally the single equation confirms the SAMAS without separately verifying the signatures.

The designed Authentication Scheme (AS) includes $\mathrm{N}$ members A1,A2, .., An, for example, $\mathrm{S}=$ $\{\mathrm{A} 1, \mathrm{~A} 2, \ldots, \mathrm{An}\}$, where the real message sender Alice is $\mathrm{A}_{\mathrm{t}}$, for several value $\mathrm{t}, 1 \leq \mathrm{t} \leq \mathrm{n}$. Let $\mathrm{x}$ is the huge prime number and $\mathrm{y}$ is the primitive element of $Z_{x}^{*}$. In addition, y is called as a generator of $Z_{x}^{*}$. Therefore $Z_{x}^{*}=(y)$. The $\mathrm{x}$ and $\mathrm{y}$ are made communal and hence pooled by all members in $\mathrm{S}$. Every $A_{i} \in S$ is a public keyg $g_{i}=y^{p i} \bmod \mathrm{x}$.

Where,

Pi- Arbitrarily chosen private key from $Z_{p-1}^{*}$.

Node $A_{i}$ and its public key $g_{i}$ is not illustrious here. The message sender has private key for transmitting message $\mathrm{m}$. The designed SAMAS is designed by using three steps.

1. Select a rapid and pairwise various $\mathrm{k}_{\mathrm{i}}$ for each $1 \leq \mathrm{i} \leq n, \mathrm{i} \neq t$ and calculate $r_{i}=y^{k_{i}} \bmod x$

2. Choose arbitrary $\mathrm{k} \in Z_{k}$ and calculate $r_{i}=y^{k} \prod_{i \neq t} g_{i}^{-r h_{i}} \bmod \mathrm{x}$ such that $r_{i} \neq 1 r_{t} \neq r_{i}$ for any $\mathrm{i} \neq \mathrm{t}$, where $\mathrm{h}_{\mathrm{i}}=\mathrm{h}\left(\mathrm{m}, \mathrm{r}_{\mathrm{i}}\right)$

Calculate $\mathrm{s}=\mathrm{k}+\sum_{i \neq 1} k_{i}+p_{1} r_{1} h_{1} \bmod (x-1)$ 
3. The SAMAS scheme for message $\mathrm{m}$ is computed as

$\mathrm{S}(\mathrm{m})=\mathrm{m}, \mathrm{S}, r_{1}, \ldots \ldots r_{n}, \mathrm{~S}$

Where,

$y^{s}=r_{1} \ldots \ldots r_{n} g_{1}^{r_{1} h_{1}} \ldots \ldots \ldots g_{n}^{r_{n} h_{n}} \bmod \mathrm{x}$ and $h_{i}=\mathrm{h}\left(\mathrm{m}, r_{i}\right)$

\section{EXPERIMENTAL RESULTS}

In this section, the performance of the proposed Secure Optimized Link State Routing (SOLSR) Protocol with FA is evaluated and compared with existing Secure Optimized Link State Routing (SOLSR) Protocol. The experiments are conducted using NS-2 simulator. The existing and proposed detection methods are compared in terms of end to end delay, energy and PDR.

\section{Performance Evaluation}

\section{End-to-end delay}

The average time taken by a packet to transmit from source to destination across the network is wellknown as End to End delay

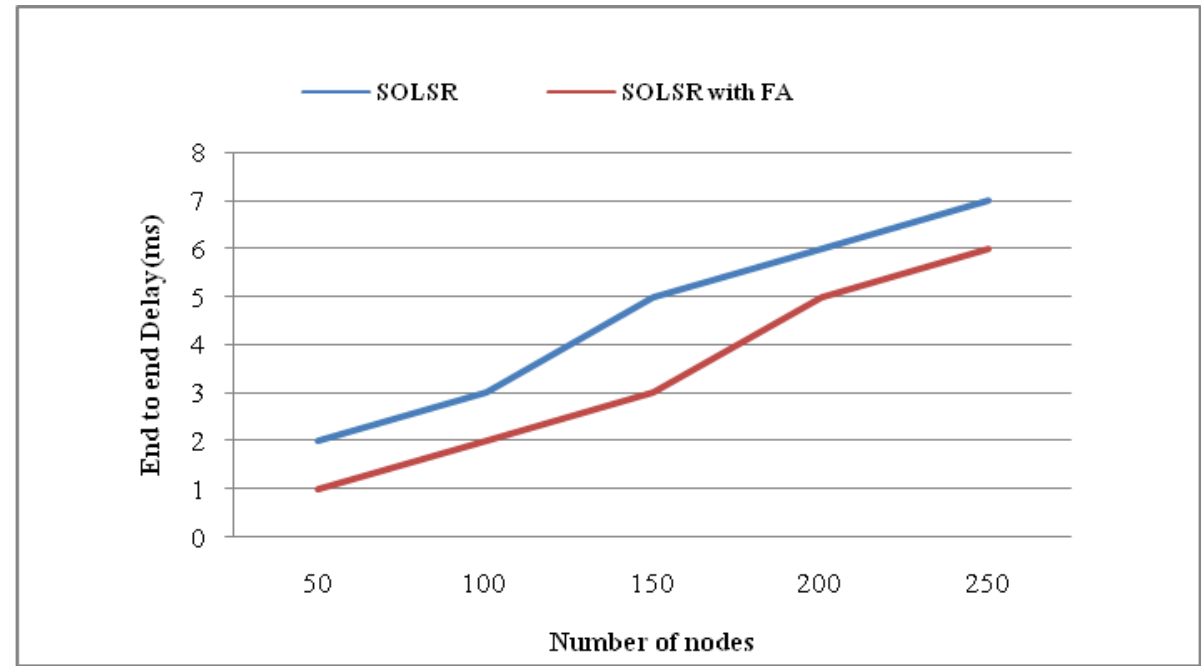

Figure.4. End-to-end delay comparison

Figure 4 shows the comparison of end to end delay performance for proposed Secure Optimized Link State Routing (SOLSR) Protocol with FA and existing Secure Optimized Link State Routing (SOLSR) Protocol. The nodes are varying from 50 to 250 and end to end delay is plotted for such nodes in milliseconds (ms). From the graph it is clear that the SOLSR with FA based achieves less end to end delay.

\section{Energy Consumption}

Energy consumption refers to the average energy necessary for transmitting, receiving or forwarding operations of a packet to a node in the network during a period of time. 
DOI: https://dx.doi.org/10.26808/rs.ca.i8v2.11 International Journal of Computer Application (2250-1797) Issue 8 Volume 2, March-April 2018

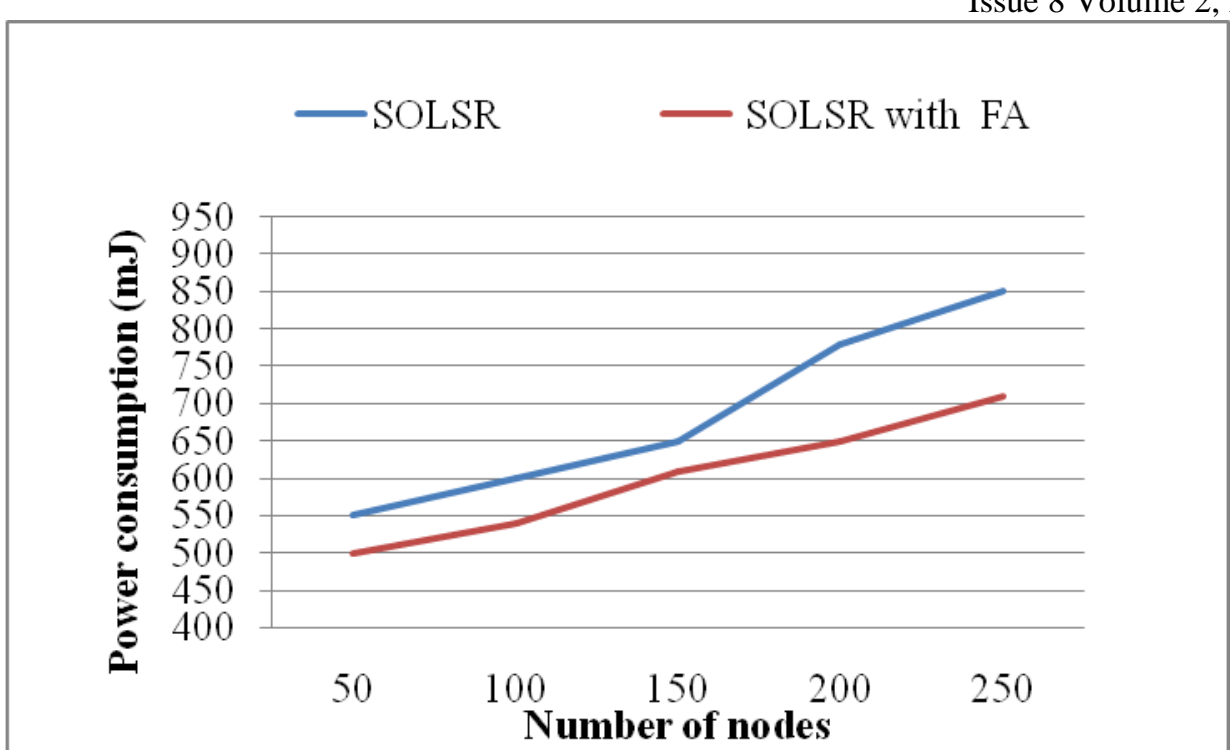

Figure 5:Energy consumption comparison

The performance of the proposed Secure Optimized Link State Routing (SOLSR) Protocol with FA is compared with the existing Secure Optimized Link State Routing (SOLSR) Protocol in terms of power consumption. In X-axis number of nodes are taken and power consumption is taken as y-axis. From the graph it is clear that the proposed SOLSR protocol provides lower energy consumption than existing method.

\section{Packet deliver ratio}

This metric is the ratio of the number of successfully delivered data packets to the destination over the total number of packets generated by all sources. High percentage of the packet delivery ratio increases network reliability and satisfies the QoS.

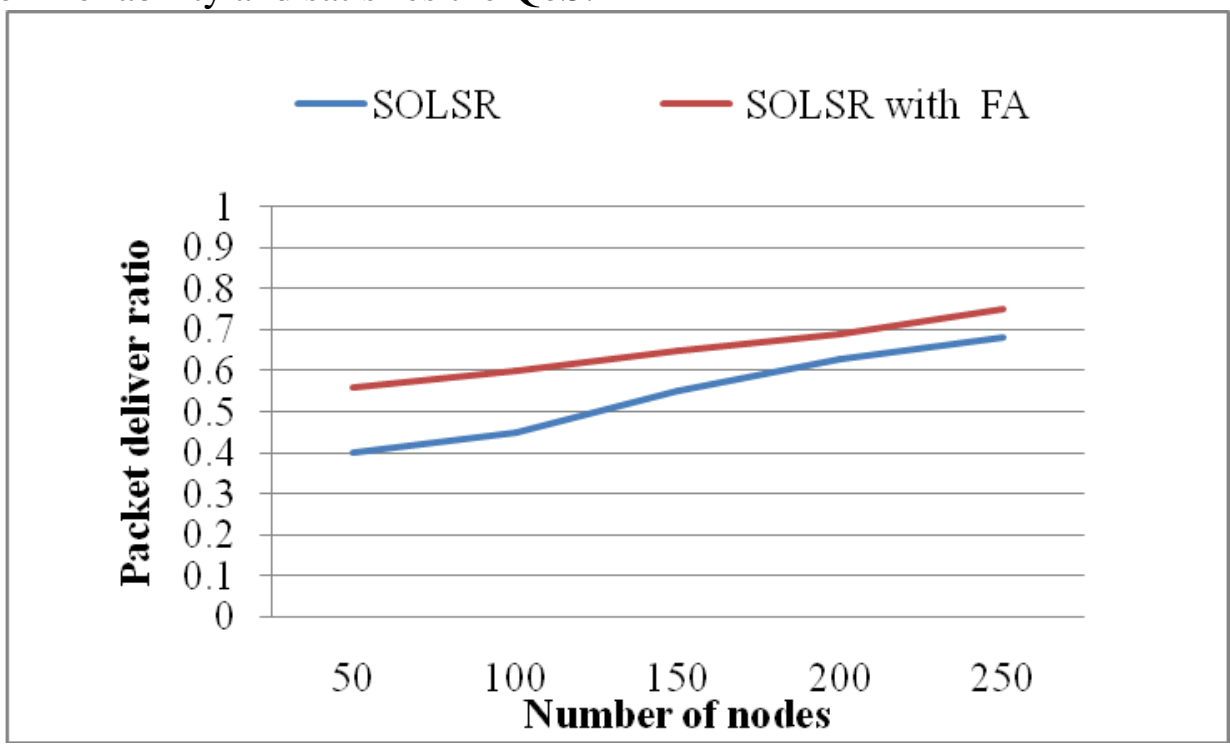

Figure.6. Packet delivery ratio comparison

Figure 6 shows the comparison of packet delivery ratio performance for proposed Secure Optimized Link State Routing (SOLSR) with FA and existing Secure Optimized Link State Routing 
DOI: https://dx.doi.org/10.26808/rs.ca.i8v2.11 International Journal of Computer Application (2250-1797)

Issue 8 Volume 2, March-April 2018

(SOLSR) Protocol. In $\mathrm{x}$-axis number of nodes is taken and packet delivery ratio is taken as $\mathrm{y}$-axis. In this work the MPRs are selected by using FA based on the packet delay and packet delivery ratio of the nodes. It improves the packet delivery ratio. From the graph it is clear that the proposed SOLSR protocol provides higher packet delivery ratio than existing method.

\section{CONCLUSION}

The proposed work describes secured transmission approach for MANETs. In this work, Secure Optimized Link State Routing (SOLSR) Protocol with Firefly Algorithm (FA) is utilized for secure and efficient packet transmission. The MPRs are selected by using FA based on the packet delay and packet deliver ratio of the nodes. In this protocol the power status of the nodes are checked and updated in routing table. Based on the node energy the routes are selected. The group key distribution is performed using the generated keys. In order to avoid non authorised nodes and use of the same group key in more than some amount of data, the group key is changed regularly. At last, the secure Source Anonymous Message Authentication Scheme (SAMAS) is performed to offer the communication privacy. The experimental results show that the proposed system attains better performance compared with the existing system in terms of PDR, energy consumption and end to end delay.

\section{REFERENCE}

1. Abusalah, L., Khokhar, A., and Guizani, M., "A survey of secure mobile Ad Hoc routing protocols," Communications Surveys \& Tutorials, IEEE, Fourth Quarter, Vol. 10, No. 4, pp. 7893, 2008.

2. Adnane, A., Bidan, C., and de Sousa Júnior, R.T. "Trust-based security for the OLSR routing protocol," Computer Communications, Vol. 36, No. 10, pp. 1159-1171, 2013.

3. Ahamad, T., and Aljumah, A., "Detection and Defense Mechanism against DDoS in MANET," Indian Journal of Science and Technology, Vol. 8, No. 33, 2015.

4. Taneja, S., \& Kush, A. (2012). Energy efficient, secure and stable routing protocol for MANET. Global Journal of Computer Science and Technology, Network Web and Security, 12(10), Version 1.0 May 2012.

5. Kong, J., Hong, X., Yi, Y., Sang Park, J., Liu, J., \& Gerla, M. (2005) A secure ad hoc routing approach using localized selfhealing communities. In Proceedings of the 6th ACM international symposium on mobile ad hoc networking and computing, pp. 254-265.

6. Kannhavong, B., Nakayama, H., Nemoto, Y., Kato, N., \& Jamalipour, A. (2007). A survey of routing attacks in mobile adhoc networks. IEEE Wireless Communications, 14(5), 85-91.

7. Chhabra, M., \& Gupta, B. B. (2014). An efficient scheme to prevent DDoS flooding attacks in mobile ad-hoc network (MANET). Research Journal of Applied Sciences, Engineering and Technology, 7(10), 2033-2039.

8. Devi, P., \& Kannammal, A. (2016). An integrated intelligent paradigm to detect DDoS attack in mobile ad hoc networks. International Journal of Embedded Systems, 8(1), 69-77.

9. Nikhil, K., Agarwal, S., and Sharma, P., "Application of Genetic Algorithm in Designing a Security Model for Mobile Adhoc Network," Department of IT, ABES Engineering College, Ghaziabad (UP) India, 2012.

10. Albers, P., Camp, O., Percher, J.M., Jouga, B., Me, L., and Puttini, R.S., "Security in Ad Hoc Networks: a General Intrusion Detection Architecture Enhancing Trust Based Approaches," Proceedings of the 1st International Workshop on Wireless Information Systems (WIS-2002), pp. 1- 12, 2002. 
DOI: https://dx.doi.org/10.26808/rs.ca.i8v2.11 International Journal of Computer Application (2250-1797) Issue 8 Volume 2, March-April 2018

11. Gupta, N.K., and Garg, A., "Trust and Shortest Path Selection based Routing Protocol for MANET," International Journal of Computer Applications (0975 - 8887), Vol. 76, No. 12, 2013.

12. Pirzada, A.A., and McDonald, C., "Deploying trust gateways to reinforce dynamic source routing," in Proceeding. 3rd International IEEE Conference on Industrial Informatics, IEEE Press, pp. 779-784, 2006. 